International Journal of Pure and Applied Mathematics

Volume 92 No. 2 2014, 225-242

ISSN: 1311-8080 (printed version); ISSN: 1314-3395 (on-line version)

url: http://www.ijpam.eu

doi: http://dx.doi.org/10.12732/ijpam.v92i2.7

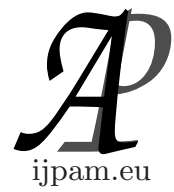

\title{
NEW CONVERGENCE METHOD WITH \\ NONMONOTONE LINE SEARCH
}

\author{
Tahar Bouali ${ }^{1}$, Yamina Laskri ${ }^{2}$ \\ ${ }^{1,2}$ Department of Mathematics \\ Badji Mokhtar University \\ Annaba 23000, ALGERIA
}

\begin{abstract}
In this paper, an efficient new nonlinear conjugate gradient method is proposed for the unconstrained optimization problems, which possesses the following property: the sufficient descent condition $g_{k}^{T} d_{k}=-\left\|g_{k}\right\|^{2}$ holds without any line search. Under the strong Wolf Non-monotone line search, we proved the global convergence of the FR method for strongly convex functions.

The numerical experiments show that the FR method is especially efficient.
\end{abstract}

AMS Subject Classification: 65K10, 90C30

Key Words: conjugate gradient, sufficient descent, non-monotone line search, global convergence, unconstrained optimization

\section{Introduction}

The first objective of this paper is to study the global convergence and practical computational performance of $F R$ conjugate gradient method with strong wolf Nonmonotone line search for nonlinear unconstrained optimization .Consider the following unconstrained optimization problem

$$
\min \left\{f(x): x \in \mathbb{R}^{n}\right\}
$$

where $f: \mathbb{R}^{n} \rightarrow \mathbb{R}$ is continuosly differentiable. The general form of the conju-

Received: December 22, 2013

(C) 2014 Academic Publications, Ltd.

$\S$ Correspondence author url: www.acadpubl.eu 
gate gradient method

$$
\begin{gathered}
x_{k+1}=x_{k}+\alpha_{k} d_{k} \\
d_{k}=\left\{\begin{array}{c}
-g_{k}, k=0 \\
-g_{k}+\beta_{k} d_{k-1}, k \geq 1
\end{array}\right.
\end{gathered}
$$

where $g_{k}=\nabla f\left(x_{k}\right), \alpha_{k}$ is a stepe-size obtained by some line search, and $\beta_{k}$ $[4,5,7,12]$ is a scalar There are many ways to select $\beta_{k}$, and some well-known formulas are given by

$$
\begin{gathered}
\beta_{k}^{P R P}=\frac{g_{k}^{T} y_{k}}{\left\|g_{k-1}\right\|^{2}} \\
\beta_{k}^{F R}=\frac{\left\|g_{k}\right\|^{2}}{\left\|g_{k-1}\right\|^{2}} \\
\beta_{k}^{C D}=\frac{\left\|g_{k}\right\|^{2}}{-d_{k-1}^{T} g_{k-1}} \\
\beta_{k}^{D Y}=\frac{\left\|g_{k}\right\|^{2}}{d_{k-1}^{T} y_{k-1}} \\
\beta_{k}^{H S}=\frac{g_{k}^{T}\left(g_{k}-g_{k-1}\right)}{d_{k-1}^{T}\left(g_{k}-g_{k-1}\right)}
\end{gathered}
$$

respectively, where $y_{k-1}=g_{k}-g_{k-1}$ and \|\| means the Euclidean norm.

In the convergence analysis and implementation of conjugate gradient method, the extended strong wolf Non-monotone line search, namely

$$
\begin{gathered}
f\left(x_{k}+\alpha_{k} d_{k}\right) \leq \max _{0 \leq j \leq m(k)} f\left(x_{k-j}\right)+\delta \alpha_{k} g_{k}^{T} d_{k} \\
\sigma_{1} g_{k}^{T} d_{k} \leq g\left(x_{k+} \alpha_{k} d_{k}\right)^{T} d_{k} \leq-\sigma_{2} g_{k}^{T} d_{k},
\end{gathered}
$$

which $0<\delta \leq \sigma_{1} \leq 1,0 \leq \sigma_{2} \leq 1,0 \leq \lambda \leq 1$ and $M_{0} \in N$,

$$
0 \leq m(k) \leq \min \left\{m(k-1)+1, M_{0}\right\}, \quad m(0)=0 .
$$

In addition, the sufficient descent condition, namely

$$
g_{k}^{T} d_{k} \leq-c\left\|g_{k}\right\|^{2}
$$

When $f$ is strongly convex, the stepsize $\alpha_{k}$ satisfies the following new nonmonotone line search condition 


$$
f\left(x_{k}+\alpha_{k} d_{k}\right)-\underset{0 \leq j \leq m(k)}{\lambda \max _{0}} f\left(x_{k-j}\right)-(1-\lambda) \min _{0 \leq j \leq m(k)} f\left(x_{k-j}\right) \leq \delta \alpha_{k} g_{k}^{T} d_{k}
$$

The convergence of (1.5),(1.6)and (1.7) with some line search condition have been studied by many authors for many years. Al-Baali [1] proved the global convergence of FR method with the strong wolf line search.Liu and al [8] and Dai and Yaun [3] extended this result to $\sigma=\frac{1}{2}$, the PRP method is generally believed to the most efficient conjugate gradient method. However, Powell [13] constructed a counter example and showed that the PRP method can circle infinitely without approaching the solution,which implies that this method is not globally convergent for general function.But the PRP $+\left(B_{k}^{P R P+}=\max \left\{0, B_{k}^{P R P}\right\}\right)$ method with the wolf line search is globally convergent when the sufficient descent condition

$$
f\left(x_{k}+\alpha_{k} d_{k}\right) \leq f\left(x_{k}\right)+\delta \alpha_{k} g_{k}^{T} d_{k}
$$

is given, the HS method is very familiar with the PRP method,the DY method with the Wolf and strong Wolf line search is globally convergent without descent condition, but the descent property of the DY method depends on line search or convexity of the objective function.

In [6], the authors propose a nonmonotone Newton method, and analyze its convergence. Lucidi and Roma [10]present a nonmonotone algorithm of FR in 1995, see [9], Liu et al in the above-montioned nonmonotonic strong Wolf line search method to prove that two types of classical conjugate gradient algorithm of the PRP method and HS method for convex objective function in this nonmonotonic Wolf line search, global convergence. In 2002, Dai Yu Hong [2] the initial nonmonotone line search method on Grippo,Lampariello and Lucidi search and hybrid conjugate DY-CD method combined with the study. In 2006 [14] on this basis, given the amendments.

In this paper we presente new global convergence of FR method conjugate gradient with nonmonotone line search type strong Wolf.

Remark. The new nonmonotone line search can be viewed as some kind of convex combination of the extended strong Wofle line search and the extended nonmonotone line search, when

$\lambda=0$ the new nonmonotone line search reduce to the extended strong Wofle line search, and when $\lambda=1$ the new nonmonotone line search reduce to the extended nonmonotone

line search. 
This paper is organized as follows. We will present a new algorithm (Algorithm 2.8), and the sufficient descent property (1.11) of Algorithm 2.8 is also given in the next section. In Section 3 the global convergence results of the $F R$ method are established.At last the preliminary numerical results are reported.

\section{New Algorithm}

Assumption 2.1. First given the general assumption of this section

$\left(\mathrm{A}_{1}\right)$ The level set $L_{1}=\left\{x \backslash f(x) \leq f\left(x_{1}\right), x \in R^{n}\right\}$ is bounded, where $x_{1}$ is the starting point

$\left(\mathrm{A}_{2}\right) f$ is strongly convex and differentiable in the level set $L_{1}$ and its gradient $g_{k}=\nabla f\left(x_{k}\right)$ lipschitz continuous . ie, there exist constants $L>0$ and $\eta>0$ making

$$
\|g(x)-g(y)\| \leq L\|x-y\|, \forall x, y \in \mathcal{N}
$$

and

$$
(g(x)-g(y))^{T}(x-y) \geq \eta\|x-y\|^{2}
$$

If $f$ satisfies Assumption $\left(\mathrm{A}_{1}\right)$ and $\left(\mathrm{A}_{2}\right)$, we can get that

$$
\|g(x)\| \leq \gamma
$$

for all $x \epsilon L_{1}$.

Now we give the following theorem, which illustrates that the formula (1.5) possesses the sufficient descent condition without any line searches.

Theorem 2.2. Consider any method (1.2) and (1.3), where $\beta_{k}=\beta_{k}^{F R}$. Then for all $k \geq 1$

$$
g_{k}^{T} d_{k}=-\left\|g_{k}\right\|^{2}
$$

Proof. Since $d_{0}=-g_{0}$, we have $g_{0}^{T} d_{0}=-\left\|g_{0}\right\|^{2}$

$$
\begin{gathered}
g_{k}^{T} d_{k}=g_{k}^{T}\left(-\theta_{k} g_{k}+\beta_{k}^{F R} d_{K-1}\right) \\
=g_{k}^{T}\left(-\frac{d_{K-1}^{T} y_{k-1}}{\left\|g_{k-1}\right\|^{2}} g_{k}+\frac{\left\|g_{k}\right\|^{2}}{\left\|g_{k-1}\right\|^{2}} d_{K-1}\right)
\end{gathered}
$$




$$
\begin{gathered}
=-\frac{d_{K-1}^{T} y_{k-1}}{\left\|g_{k-1}\right\|^{2}}\left\|g_{k}\right\|^{2}+\frac{\left\|g_{k}\right\|^{2}}{\left\|g_{k-1}\right\|^{2}} g_{k}^{T} d_{K-1} \\
=\frac{\left\|g_{k}\right\|^{2}}{\left\|g_{k-1}\right\|^{2}}\left(-d_{K-1}^{T} y_{k-1}+g_{k}^{T} d_{K-1}\right) \\
=\frac{\left\|g_{k}\right\|^{2}}{\left\|g_{k-1}\right\|^{2}}\left(-d_{K-1}^{T} y_{k-1}+d_{K-1}^{T} g_{k}\right) \\
=\frac{\left\|g_{k}\right\|^{2}}{\left\|g_{k-1}\right\|^{2}}\left[-d_{K-1}^{T}\left(g_{k}-g_{k-1}\right)+d_{K-1}^{T} g_{k}\right] \\
=\frac{\left\|g_{k}\right\|^{2}}{\left\|g_{k-1}\right\|^{2}} d_{K-1}^{T} g_{k-1}
\end{gathered}
$$

Thus, we find a recurrence relation

$$
g_{k}^{T} d_{k}=\frac{\left\|g_{k}\right\|^{2}}{\left\|g_{k-1}\right\|^{2}} d_{K-1}^{T} g_{k-1}
$$

so

$$
\begin{aligned}
g_{k}^{T} d_{k} & =\frac{\left\|g_{k}\right\|^{2}}{\left\|g_{k-1}\right\|^{2}} d_{K-1}^{T} g_{k-1} \\
& =\frac{\left\|g_{k}\right\|^{2}}{\left\|g_{k-1}\right\|^{2}} \frac{\left\|g_{k-1}\right\|^{2}}{\left\|g_{k-2}\right\|^{2}} d_{K-2}^{T} g_{k-2}=\frac{\left\|g_{k}\right\|^{2}}{\left\|g_{k-2}\right\|^{2}} d_{K-2}^{T} g_{k-2} \\
& =\cdots \\
& =\frac{\left\|g_{k}\right\|^{2}}{\left\|g_{0}\right\|^{2}} d_{0}^{T} g_{0} \\
& =\frac{\left\|g_{k}\right\|^{2}}{\left\|g_{0}\right\|^{2}}\left(-\left\|g_{0}\right\|^{2}\right)=-\left\|g_{k}\right\|^{2}<0
\end{aligned}
$$

Lemma 2.3. Suppose that assumption 2.1 holds and $\alpha_{k}$ is obtainded by the naw Nonmonotone line search(1.12) and (1.10). Then there exist $c_{1}>0$, such that

$$
\left\|s_{k}\right\| \geq \frac{c_{1}\left(-g_{k}^{T} d_{k}\right)}{\left\|d_{k}\right\|^{2}}
$$

where $s_{k}=x_{k+1}-x_{k}$. 
Proof. From assumption $2.1\left(\mathrm{~A}_{1}\right)$

$$
\|g(x)-g(y)\| \leq L\|x-y\|
$$

that is

$$
\left\|y_{k}\right\| \leq L\left\|s_{k}\right\|
$$

so we have

$$
y_{k}^{T} d_{k} \leq L\left\|s_{k}\right\|\left\|d_{k}\right\|
$$

and from (1.10) we can get

$$
y_{k}^{T} d_{k}=d_{k}^{T}\left(g_{k+1}-g_{k}\right) \geq\left(1-\sigma_{1}\right)\left(-g_{k}^{T} d_{k}\right)
$$

Thus, from (2.7) and (2.8) we obtain

$$
\left\|s_{k}\right\| \geq \frac{\left(1-\sigma_{1}\right)}{L} \times \frac{\left(-g_{k}^{T} d_{k}\right)}{\left\|d_{k}\right\|^{2}}
$$

let $c_{1}=\frac{\left(1-\sigma_{1}\right)}{L}$ and from (2.9), we obtain (2.5). Therefore, our proof is complete.

Lemma 2.4. Suppose that assumption 2 holds, $\alpha_{k}$ is given (1.12) and (1.10), and $\beta_{k}=\beta_{k}^{F R}$. Then there exist a positive constant $M=\frac{L^{2}}{\eta}$ such that

$$
\frac{\left\|y_{k}\right\|^{2}}{y_{k}^{T} d_{k}} \leq M
$$

Proof. By the convexity, assumption we have

$$
y_{k}^{T} d_{k}=d_{k}^{T}\left(g_{k+1}-g_{k}\right) \geq \eta \alpha_{k}\left\|d_{k}\right\|^{2}
$$

and from the lipschitz continuity (2.1), we can get that

$$
\left\|y_{k}\right\|=\left\|g_{k+1}-g_{K}\right\| \leq L\left\|x_{k+1}-x_{k}\right\|=L \alpha_{k}\left\|d_{k}\right\|
$$

Utilising (2.11) and (2.12), we can get that

$$
\frac{\left\|y_{k}\right\|^{2}}{y_{k}^{T} d_{k}} \leq \frac{L^{2} \alpha_{k}^{2}\left\|d_{k}\right\|^{2}}{\eta \alpha_{k}^{2}\left\|d_{k}\right\|^{2}}=\frac{L^{2}}{\eta}=M
$$

which completes the proof. 
Lemma 2.5. Assume that the following inequality holds for all $k$

$$
0<m_{1} \leq\left\|g_{k}\right\| \leq m_{2}
$$

and $\alpha_{k}$ is given by (1.12) and (1.10), then:

(1) there exist a positive constant $b>1$ such that

$$
\left|\beta_{k}\right| \leq b
$$

(2) there exist a positive constant $\lambda$, when $\left\|y_{k-1}\right\| \leq \lambda$, we have $\left|\beta_{k}\right| \leq \varepsilon$ for any $\varepsilon>0$.

Proof. When $\beta_{k}=\beta_{k}^{F R}$, from (2.4) and (2.14) we can get

$$
\begin{aligned}
\left|\beta_{k}\right| & =\frac{\left\|g_{k}\right\|^{2}}{\left\|g_{k-1}\right\|^{2}}=\frac{\left\|g_{k}-g_{k-1}+g_{k-1}\right\|^{2}}{\left\|g_{k-1}\right\|^{2}} \\
& =\frac{\left\|g_{k}-g_{k-1}+g_{k-1}\right\|\left\|g_{k}-g_{k-1}+g_{k-1}\right\|}{\left\|g_{k-1}\right\|^{2}} \\
& \leq \frac{\left.\left\|g_{k}-g_{k-1}\right\|+\left\|g_{k-1}\right\|\right)\left(\left\|g_{k}-g_{k-1}\right\|+\left\|g_{k-1}\right\|\right)}{\left\|g_{k-1}\right\|^{2}} \\
& =\frac{\left\|g_{k}-g_{k-1}\right\|^{2}+2\left\|g_{k}-g_{k-1}\right\|\left\|g_{k-1}\right\|+\left\|g_{k-1}\right\|^{2}}{\left\|g_{k-1}\right\|^{2}} \\
& \leq \frac{\left\|g_{k}\right\|^{2}+\left\|g_{k-1}\right\|^{2}+2\left\|g_{k}\right\|\left\|g_{k-1}\right\|+2\left\|g_{k}-g_{k-1}\right\|\left\|g_{k-1}\right\|+\left\|g_{k-1}\right\|^{2}}{\left\|g_{k-1}\right\|^{2}} \\
& \leq \frac{\left\|g_{k}\right\|^{2}+\left\|g_{k-1}\right\|^{2}+2\left\|g_{k}\right\|\left\|g_{k-1}\right\|+2\left\{\left\|g_{k}\right\|+\left\|g_{k-1}\right\|\right\}\left\|g_{k-1}\right\|+\left\|g_{k-1}\right\|^{2}}{\left\|g_{k-1}\right\|^{2}} \\
& =\frac{\left\|g_{k}\right\|^{2}+2\left\|g_{k-1}\right\|^{2}+2\left\|g_{k}\right\|\left\|g_{k-1}\right\|+2\left\|g_{k}\right\|\left\|g_{k-1}\right\|+2\left\|g_{k-1}\right\|^{2}}{\left\|g_{k-1}\right\|^{2}} \\
& \leq \frac{9 g_{k}^{2}\left\|^{2}+4\right\| g_{k-1}\left\|^{2}+4\right\| g_{k}\|\| g_{k-1} \|}{m_{1}^{2}}=\left(\frac{3 m_{2}}{m_{1}}\right)^{2} \cdot
\end{aligned}
$$

Let $b=\left(\frac{3 m_{2}}{m_{1}}\right)^{2}$,obviously we have $b>1$ because of $m_{2} \geq m_{1}>0$. 
Let $\lambda=\frac{2 m_{1}^{3}}{9 m_{2}^{2}} \varepsilon$

$$
\begin{aligned}
\left|\beta_{k}\right| & =\frac{\left\|g_{k}\right\|^{2}}{\left\|g_{k-1}\right\|^{2}} \leq \frac{\left\|g_{k}-g_{k-1}\right\|^{2}+2\left\|g_{k}-g_{k-1}\right\|\left\|g_{k-1}\right\|+\left\|g_{k-1}\right\|^{2}}{\left\|g_{k-1}\right\|^{2}} \\
& \leq \frac{\left\|g_{k}-g_{k-1}\right\|^{2}+2\left\|g_{k}-g_{k-1}\right\|\left\|g_{k-1}\right\|-g_{k-1}^{T} d_{k-1}}{\left\|g_{k-1}\right\|^{2}} \\
& \leq \frac{\left\|g_{k}-g_{k-1}\right\|^{2}+2\left\|g_{k}-g_{k-1}\right\|\left\|g_{k-1}\right\|+g_{k-1}^{T} d_{k-1}}{\left\|g_{k-1}\right\|^{2}} \\
& \leq \frac{\left\|g_{k}-g_{k-1}\right\|^{2}+2\left\|g_{k}-g_{k-1}\right\|\left\|g_{k-1}\right\|}{\left\|g_{k-1}\right\|^{2}}+\frac{g_{k-1}^{T} d_{k-1}}{\left\|g_{k-1}\right\|^{2}}
\end{aligned}
$$

we have

$$
g_{k-1}^{T} d_{k-1}<0
$$

Therfore

$$
\begin{aligned}
\left|\beta_{k}\right| & \leq \frac{\left\|g_{k}-g_{k-1}\right\|^{2}+2\left\|g_{k}-g_{k-1}\right\|\left\|g_{k-1}\right\|}{\left\|g_{k-1}\right\|^{2}} \\
& =\frac{\left\|g_{k}-g_{k-1}\right\|\left(\left\|g_{k}-g_{k-1}\right\|+2\left\|g_{k-1}\right\|\right)}{\left\|g_{k-1}\right\|^{2}} \\
& \leq \frac{\left(\left\|g_{k}\right\|+3\left\|g_{k-1}\right\|\right)\left\|y_{k-1}\right\|}{\left\|g_{k-1}\right\|^{2}} \\
& =\frac{4 m_{2}}{m_{1}^{2}}\left\|y_{k-1}\right\|=\frac{8 m_{2}^{2}}{2 m_{1}^{2} m_{2}}\left\|y_{k-1}\right\| \\
& \leq \frac{8 m_{2}^{2}}{2 m_{1}^{2} m_{1}}\left\|y_{k-1}\right\| \leq \frac{9 m_{2}^{2}}{2 m_{1}^{3}}\left\|y_{k-1}\right\| \\
& \leq \frac{9 m_{2}^{2}}{2 m_{1}^{3}} \lambda \leq \varepsilon
\end{aligned}
$$

This completes the proof.

Lemma 2.6. Suppose that assumption $2.1\left(A_{1}\right)$ holds and $\alpha_{k}$ is obtained by the new Nonmonotone line search(1.12) and (1.10), denote $\zeta_{k}=\delta \alpha_{k} g_{k}^{T} d_{k}$, then $\left\{f\left(x_{k}\right)\right\}$ is nonincreasing and

$$
\lim _{k \rightarrow \infty} \zeta_{l(k+1)-1}=0
$$


where

$$
l(k)=\max \left\{i \mid 0 \leq k-i \leq m(k), f\left(x_{i}\right)=\max _{0 \leq j \leq m<k} f\left(x_{k}-j\right)\right\}
$$

and $k-m(k) \leq l(k) \leq(k)$.

Proof. From (1.12) and (2.17) we can get that

$$
\begin{gathered}
f\left(x_{k}+\alpha_{k} d_{k}\right) \leq \operatorname{mix}_{0 \leq j \leq m(k)}^{\lambda} f\left(x_{k-j}\right)+(1-\lambda) \min _{0 \leq j \leq m(k)} f\left(x_{k-j}\right)+\delta \alpha_{k} g_{k}^{T} \\
\leq f\left(x_{l(k)}\right)+\zeta_{k}
\end{gathered}
$$

because $\zeta_{k}<0$,we can obtain

$$
f\left(x_{(k+1)}\right) \leq f\left(x_{l(k)}\right.
$$

from (2.17) and

$$
m(k) \leq m(k-1)+1
$$

for all $k$, we can get that

$$
\begin{gathered}
f\left(x_{l(k)}\right) \leq \max _{0 \leq j \leq m(k-1)+1} f\left(x_{k-j}\right)=\max \left\{\max _{0 \leq j \leq m(k-1)} f\left(x_{k-1-j}\right), f\left(x_{k}\right)\right\} \\
=\max f\left(x_{l(k-1)}\right), f\left(x_{k}\right)=f\left(x_{l(k-1),} \quad k=1,2, \ldots\right.
\end{gathered}
$$

from the above ineaquation and (2.19) we can obtain

$$
f\left(x_{l(k+1)}\right) \leq f\left(x_{l(l(k+1)-1)}\right)+\zeta_{l(k+1)-1}
$$




$$
\leq f\left(x_{l\left(k-M_{0}\right)}\right)+\zeta_{l(k+1)-1}
$$

hence, we have

$$
0 \leq-\zeta_{l(k+1)-1} \leq f\left(x_{l\left(k-M_{0}\right)}\right)-f\left(x_{l(k+1)}\right)
$$

by $(2.22)$ when assumption $2.1\left(\mathrm{~A}_{1}\right)$ holds, we have

$$
\lim _{k \rightarrow \infty} \zeta_{l(k+1)-1}=0
$$

Lemma 2.7. Suppose that $\alpha_{k}$ is given by (1.12) and (1.10) and $\beta_{k}=\beta_{k}^{F R}$. Then $\{l(k)\}$ is increasing

Proof. . Assume that

$$
l(k+1)<l(k)
$$

then we have

$$
k+1 \geq k \geq l(k)>l(k+1) \geq k+1-m(k+1)
$$

by the definition of $l(k+1)$ and (2.20), we can obtain

$$
f\left(x_{l(k+1)}\right) \geq f\left(x_{l(k)}\right)
$$

but from the lemma 2.6 we have

$$
f\left(x_{l(k+1)}\right) \leq f\left(x_{l(k)}\right)
$$

hence,by the definition of $l(k+1)$ and (2.25), we have

$$
l(k+1) \geq l(k)
$$

which is contradictory to $(2.25)$. Hence $l(k) \leq l(k+1)$, namely $\{l(k)\}$ is increasing

Now we can present a new descent conjugate gradient method as follows:

\section{$F R$ method with nonmonotone line search (algorithm)}

Stepe 1 : Given $x_{1} \in \mathbb{R}^{n}, \varepsilon \geq 0,0<\delta \leq \sigma_{1} \leq 1,0 \leq \sigma_{2} \leq 1, M_{0} \in N, 0 \leq$ $\lambda \leq 1$

set $d_{1}=-g_{1}, k=1$, if $\left\|g_{k}\right\| \leq \varepsilon$, then stop. 
Stepe 2 : Find $\alpha_{k} \geq 0$ by (1.12) and (1.10).

Stepe 3 : Let $x_{k+1}=x_{k}+\alpha_{k} d_{k}$ and $g_{k+1}=g\left(x_{k+1}\right)$,if $\left\|g_{k+1}\right\| \leq \varepsilon$, then stop.

Stepe 4 : Compute $\beta_{k}=\beta_{k}^{F R}$ by the formula (1.5) and generate $d_{k+1}$ by (1.3).

Stepe 5 : Set $k=k+1$,go to step2.

\section{Global Convergence}

Theorem 3.1. Suppose that assumption holds and $\alpha_{k}$ is obtained by the new Nonmonotone line search (1.12) and (1.10), consider any iteration method of the form (1.2) and (1.3), where $\beta_{k}=\beta_{k}^{F R}$. Then

$$
\lim _{k \rightarrow \infty} \inf \left\|g_{k}\right\|=0
$$

Proof. . If assumption 2.1, then there exists a constant $\gamma_{1}$ such that

$$
0<\gamma_{1} \leq\|g(x)\| \leq \gamma_{2}
$$

for all $k$. From (1.3) and lemma 2.5 we have

$$
\begin{array}{rlr}
\left\|d_{l(k+1)-1}\right\| & & \\
& \leq & \\
& \leq & g_{l(k+1)-1}\left\|+\left|\beta_{l(k+1)-2}\right|\right\| d_{l(k+1)-2} \| \\
& \leq & \gamma_{2}+b\left\|d_{l(k+1)-2}\right\| \\
\cdots & \sum_{j=0}^{l(k+1)-l(k)-3}(b)^{j}+(b)^{l(k+1)-l(k)-2}\left\|d_{l(k)+1}\right\| \\
& \leq \gamma_{2} \sum_{j=0}^{l(k+1)-l(k)-2}(b)^{j}+(b)^{l(k+1)-l(k)-2}\left|\beta_{l(k)+1}\right|\left\|d_{l(k)}\right\|
\end{array}
$$

because

$$
l(k+1)-l(k) \leq k+1-[k-m(k)] \leq M_{0}+1
$$

we can get

$$
\bar{\gamma} \sum_{j=0}^{l(k+1)-l(k)-2}(b)^{j} \leq \sum_{j=0}^{M_{0}}(b)^{j},\left(b^{\prime}\right)^{l(k+1)-l(k)-2} \leq(b)^{M_{0}}
$$


where

$$
\gamma_{2} \sum_{j=0}^{M_{0}}(b)^{j}=h_{1},(b)^{M_{0}}=h_{2}
$$

Then

$$
\left\|d_{l(k+1)-1}\right\| \leq h_{1}+h_{2}\left|\beta_{l(k)}\right|\left\|d_{l(k)}\right\|
$$

from lemma 2.3and (1.10) we have

$$
0 \leq\left\|y_{k}\right\| \leq \sqrt{M y_{k}^{T} s_{k}} \leq \sqrt{M\left(1+\sigma_{2}\right)\left(-\alpha_{k} g_{k}^{T} d_{k}\right)}
$$

from lemma 2.5 we not that $\varepsilon=\frac{1}{h_{2} b^{2}}$, there exists an innegative integer $k_{0}$ when $k \geq k_{0}$ we have

$\left|\beta_{l(k)}\right|<\varepsilon$

Thus we can get

$$
\begin{aligned}
\left\|d_{l(k+1)-1}\right\| & \leq h_{1}+\frac{\left\|d_{l(k)}\right\|}{b^{2}} \\
& \leq h_{1}+\frac{\left\|g_{l(k)}\right\|+\left|\beta_{l(k)}\right|\left\|d_{l(k)-1}\right\|}{b^{2}} \\
& \leq h_{1}+\frac{\gamma_{2}+b\left\|d_{l(k)-1}\right\|}{b^{2}} \\
& \leq h_{3}+\frac{\left\|d_{l(k)-1}\right\|}{b}
\end{aligned}
$$

where $h_{3}=h_{1}+\frac{\gamma_{2}}{b^{2}}$, we have a recursive equation which leads to

$$
\begin{aligned}
\left\|d_{l(k+1)-1}\right\| & \leq h_{3}+\frac{\left\|d_{l(k)-1}\right\|}{b} \\
& \leq h_{3}+\frac{h_{3}+\frac{\left\|d_{l(k-1)-1}\right\|}{b} \leq \ldots}{b} \leq h_{3} \sum_{j=0}^{k-k_{0}}\left(\frac{1}{b}\right)^{j}+\frac{1^{k-k_{0}+1}}{b}\left\|d_{\imath\left(k_{0}\right)-1}\right\| \\
& \leq h_{3} \sum_{j=0}^{\infty}\left(\frac{1}{b}\right)^{j}+\left\|d_{\imath\left(k_{0}\right)-1}\right\|
\end{aligned}
$$




$$
\leq h_{3} \frac{b}{b-1}+\left\|d_{\imath\left(k_{0}\right)-1}\right\|
$$

Applying $l(k) \geq k-m(k) \geq k-M_{0}$ and lemma 2.7 we can assume

$$
l(i)-1 \leq j<l(i+1)-1, i \geq k_{0}+2
$$

for all $j \geq l\left(k_{0}+2\right)-1$, thus we have

$$
\begin{aligned}
&\left\|d_{j}\right\| \leq\left\|g_{j}\right\|+\left|\beta_{j}\right|\left\|d_{j}-1\right\| \leq \gamma_{2}+b\left(\left\|g_{j-1}\right\|+\left|\beta_{j-1}\right|\left\|d_{j}-1\right\|\right) \leq \ldots \\
& \leq \\
& \gamma_{2} \sum_{t=0}^{j-l(i)}(b)^{t}+(b)^{j-l(i)+1}\left\|d_{l(i)-1}\right\|
\end{aligned}
$$

Therefore, from

$$
\begin{aligned}
j-l(i)+1 & \leq[l(i+1)-1]-l(i)+1 \\
& \leq i+1-[i-m(i)] \\
& \leq M_{0}+1
\end{aligned}
$$

and (3.5), we have

$$
\left\|d_{j}\right\| \leq \gamma_{2} \sum_{t=0}^{j-l(i)}\left(b^{t}+(b)^{M_{0}+1}\left\|d_{l(i)-1}\right\|\right.
$$

From (3.4) and (3.6), we have

$$
\left\|d_{j}\right\| \leq \gamma_{2} \sum_{t=0}^{M_{0}}(b)^{t}+(b)^{M_{0}+1}\left[h_{3} \frac{b}{b-1}+\left\|d_{\imath\left(k_{0}\right)-1}\right\|\right]
$$

for all $i-1>k_{0}$. By using lemma 2.3 and lemma 2.4 we have

$$
\begin{aligned}
&-\zeta_{l(k+1)-1}=\frac{\rho\left\|s_{l(k+1)-1}\right\|\left(-g_{l(k+1)-1}, d_{l(k+1)-1}\right)}{\left\|d_{l(k+1)-1}\right\|} \\
& \geq \rho c_{1} \frac{\left(-g_{l(k+1)-1}, d_{l(k+1)-1}\right)^{2}}{\left\|d_{l(k+1)-1}\right\|} \\
& \geq \rho c_{1} \frac{\gamma_{1}^{4}}{\left\|d_{l(k+1)-1}\right\|} \geq 0
\end{aligned}
$$

but from (2.16) and (3.8) we can get 


\begin{tabular}{|c|c|}
\hline Problems & Name \\
\hline 1 & Roth function \\
\hline 2 & Beale function \\
\hline 3 & Powellevalley function \\
\hline 4 & Wood function \\
\hline 5 & Kowalikand Osborne function \\
\hline 6 & Brownand Dennis function \\
\hline 7 & Watson function \\
\hline 8 & Etended Rosenbrock function \\
\hline 9 & Trigonometric function \\
\hline 10 & Extended Powell singular function \\
\hline 11 & Penalty functionI \\
\hline 12 & Penalty functionII \\
\hline 13 & \\
\hline 14 & \\
\hline
\end{tabular}

Table 4.1: List of test problems

$$
\lim _{k \rightarrow \infty} \frac{1}{\left\|d_{l(k+1)-1}\right\|}=0
$$

which is contradictory to $(3.7)$

$$
\lim _{k \rightarrow \infty} \inf \left\|g_{k}\right\|=0
$$

\section{Numerical Experiments}

We performed some numerical experiments on conjugate methods with the new nonmonotone line search type strong Wolf. We tested the FR method,,DY method, HS method and PRP method.the methods were tested from [11], when $\varepsilon=10^{-6}, \delta=0.01, \sigma_{1}=\sigma_{2}=0.1, \lambda=0, M_{0}=150$ for when $\varepsilon=10^{-6}$, $\delta=0.01, \sigma_{1}=\sigma_{2}=0.1, \lambda=\frac{1}{2}, M_{0}=150$.The tested problems are listed in Table 4.1, and the detail numerical results of our tests are reported in Tables 4.2, the detailed numerical results are listed in the form NI/NF/NG, where NI,NF,NG denote the number of iterations, Function evaluations and gradient.

The star $(*)$ denotes that this result is best one among these methods. In table 4.3, CPU times of the methods are given. Tables 4.2 and 4.3 show that the 


\begin{tabular}{|c|c|c|c|c|c|}
\hline Probl. & Dimen. & $\mathrm{FR}$ & DY & HS & PRP \\
\hline 1 & 2 & $11 / 70 / 56^{*}$ & $42 / 168 / 139$ & $55 / 187 / 155$ & $11 / 76 / 57$ \\
\hline 2 & 2 & $14 / 57 / 45$ & $75 / 186 / 164$ & $74 / 177 / 155$ & $13 / 58 / 45^{*}$ \\
\hline 3 & 3 & $40 / 148 / 123$ & $37 / 118 / 98^{*}$ & $56 / 157 / 132$ & $66 / 183 / 156$ \\
\hline 4 & 3 & $1 / 2 / 2^{*}$ & $1 / 2 / 2^{*}$ & $1 / 2 / 2^{*}$ & $1 / 2 / 2^{*}$ \\
\hline 5 & 4 & $103 / 333 / 383$ & $2286 / 4555 / 4547$ & $425 / 1036 / 947$ & $113 / 380 / 328$ \\
\hline 6 & 4 & $78 / 278 / 230^{*}$ & $100 / 291 / 240$ & $184 / 438 / 399$ & $118 / 357 / 304$ \\
\hline 7 & 4 & $68 / 249 / 220$ & $536 / 1449 / 1271$ & $254 / 723 / 633$ & $93 / 269 / 240$ \\
\hline 8 & $\frac{1}{5}$ & $41 / 178 / 136$ & $39 / 158 / 121$ & $44 / 171 / 133$ & $37 / 156 / 123^{*}$ \\
\hline 9 & $\begin{array}{c}5 \\
20\end{array}$ & $\begin{array}{c}403 / 1210 / 1062 \\
2287 / 7972 / 7045\end{array}$ & $\begin{array}{c}126 / 348 / 298 \\
1945 / 5658 / 4924\end{array}$ & $\begin{array}{c}87 / 276 / 238^{*} \\
4375 / 12695 / 11223\end{array}$ & $\begin{array}{c}133 / 374 / 338 \\
3293 / 10458 / 9246\end{array}$ \\
\hline 10 & $\begin{array}{l}100 \\
200\end{array}$ & $\begin{array}{c}33 / 195 / 152 \\
24 / 167 / 125^{*}\end{array}$ & $\begin{array}{l}31 / 157 / 121 \\
26 / 160 / 121\end{array}$ & $\begin{array}{l}62 / 223 / 182 \\
25 / 159 / 117\end{array}$ & $\begin{array}{c}29 / 168 / 128^{*} \\
25 / 175 / 132\end{array}$ \\
\hline 11 & $\begin{array}{l}100 \\
200\end{array}$ & $\begin{array}{c}56 / 137 / 120^{*} \\
64 / 168 / 140\end{array}$ & $\begin{array}{l}306 / 401 / 401 \\
314 / 399 / 398\end{array}$ & $\begin{array}{l}F A I L \\
F A I L\end{array}$ & $\begin{array}{l}59 / 120 / 114 \\
64 / 135 / 127\end{array}$ \\
\hline 12 & $\begin{array}{c}500 \\
1000\end{array}$ & $\begin{array}{c}219 / 490 / 465^{*} \\
38 / 69 / 64^{*}\end{array}$ & $\begin{array}{c}4796 / 6823 / 6822 \\
414 / 449 / 449\end{array}$ & $\begin{array}{l}5089 / 7049 / 7058 \\
2406 / 3114 / 3115\end{array}$ & $\begin{array}{c}1645 / 2889 / 2889 \\
147 / 251 / 252\end{array}$ \\
\hline 13 & $\begin{array}{c}500 \\
1000\end{array}$ & $\begin{array}{c}6 / 13 / 7^{*} \\
7 / 14 / 8\end{array}$ & $\begin{array}{l}7 / 16 / 8 \\
7 / 15 / 8\end{array}$ & $\begin{array}{l}7 / 16 / 8 \\
7 / 15 / 8\end{array}$ & $\begin{array}{c}7 / 16 / 8 \\
6 / 13 / 7^{*}\end{array}$ \\
\hline 14 & $\begin{array}{l}1000 \\
5000\end{array}$ & $\begin{array}{l}33 / 75 / 60^{*} \\
32 / 75 / 60^{*}\end{array}$ & $\begin{array}{l}52 / 107 / 102 \\
65 / 137 / 131\end{array}$ & $\begin{array}{l}52 / 114 / 109 \\
72 / 149 / 145\end{array}$ & $\begin{array}{l}36 / 78 / 75 \\
35 / 76 / 73\end{array}$ \\
\hline
\end{tabular}

Table 4.2: The numerical results of the methods

FR method has the best performance with respect to the number of iterations and CPU time.All numerical results show that the efficiency of the FR method is encouraging

In order to rank the iterative numerical methods, one can compute the total nSumber of function and gradient evaluations by the formula

$$
N_{\text {total }}=N F+5 \times N G
$$

Similarly, we compare $P R P$ method, $H S$ method, $D Y$ method with $F R$ method as follows: for each problem $i$, compute the total numbers of function evaluations and gradient evaluations required by the evaluated methods and $F R$ method by formula (4.1), and denote them by $N_{\text {total, } i}(\mathrm{EM})$ and $N_{\text {total,i }}$ $(\mathrm{FR})$; then calculate the ratio

$$
r_{i}[E M(J)]=\frac{N_{\text {total, } i}[E M(J)]}{N_{\text {total, } i}(F R)}
$$

If $\left[E M\left(J_{0}\right)\right]$ method does not work for example $i_{0}$, but $F R$ method can work, we replace the $r_{i_{0}}\left[E M\left(J_{0}\right)\right]$ by a positive constant $\tau_{1}$ which define as follows:

$$
\tau_{1}=\max \left\{r_{i}\left[E M\left(J_{0}\right)\right]:\left(i, j_{0}\right) \in S_{1}\right\}
$$

where

$$
S_{1}=\left\{\left(i, j_{0}\right): \text { method } j_{0} \text { does not work for example } i\right\}
$$

If $F R$ method does not work for example $i_{0}$, but [EM $\left.\left(J_{0}\right)\right]$ method can work, we replace the $r_{i_{0}}\left[E M\left(J_{0}\right)\right]$ by a positive constant $\tau_{2}$ which define as 


\begin{tabular}{|c|c|c|c|c|c|}
\hline Problems & dimension & FR & DY & HS & PRP \\
\hline 1 & 2 & $0.0451^{*}$ & 0.1775 & 0.1964 & 0.0516 \\
\hline 2 & 2 & 0.0721 & 0.3100 & 0.2717 & $0.0713^{*}$ \\
\hline 3 & 3 & 0.2530 & $0.1264^{*}$ & 0.1995 & 0.5374 \\
\hline 4 & 3 & $0.0008^{*}$ & 0.0041 & 0.0044 & 0.0036 \\
\hline 5 & 4 & 0.3892 & 8.9351 & 2.5667 & 0.7034 \\
\hline 6 & 4 & $0.3788^{*}$ & 0.4389 & 0.7155 & 0.5266 \\
\hline 7 & 4 & 0.3570 & 2.1098 & 0.6122 & 0.4177 \\
\hline 8 & 5 & 0.2364 & 0.3521 & $0.1614^{*}$ & 0.2172 \\
\hline \multirow{2}{*}{9} & 5 & 2.2057 & 0.5362 & $0.3141^{*}$ & 0.6104 \\
& 20 & 7.5890 & $6.100^{*}$ & 16.9904 & 10.9497 \\
\hline \multirow{2}{*}{10} & 100 & $0.2136^{*}$ & 0.2332 & 0.5122 & 0.2718 \\
& 200 & $0.3657^{*}$ & 0.4020 & 0.3961 & 0.4309 \\
\hline \multirow{2}{*}{11} & 100 & $0.3899^{*}$ & 1.9000 & $F A I L$ & 0.4573 \\
& 200 & 1.9860 & 6.5000 & $F A I L$ & $1.8896^{*}$ \\
\hline \multirow{2}{*}{12} & 500 & $8.7861^{*}$ & 56.4843 & 57.1386 & 28.8092 \\
& 1000 & $1.8963^{*}$ & 15.0000 & 40.6372 & 6.1500 \\
\hline \multirow{2}{*}{13} & 500 & 1.9425 & 2.1589 & 2.1428 & $1.8554^{*}$ \\
& 1000 & 8.3452 & 9.7060 & 8.5487 & $7.3760^{*}$ \\
\hline \multirow{2}{*}{14} & 1000 & $0.5001^{*}$ & 0.6897 & 0.7284 & 0.5066 \\
& 5000 & $1.3960^{*}$ & 3.1576 & 3.6011 & 1.7479 \\
\hline
\end{tabular}

Table 4.3: The corresponding CPU times of the methods

follows:

$$
\tau_{2}=\min \left\{r_{i}\left[E M\left(J_{0}\right)\right]:\left(i, j_{0}\right) \in S_{1}\right\}
$$

Neither FR method nor $\left[E M\left(J_{0}\right)\right]$ method works, we define $r_{i_{0}}\left[E M\left(J_{0}\right)\right]=$ 1.The geometric mean of these ratios for $[E M(J)]$ method over all the test problems is defined by

$$
r[E M(J)]=\left\{\Pi_{i \in s} r_{i}[E M(J)]\right\}^{\frac{1}{|S|}}
$$

where $S$ denotes the set of the test problems and $|S|$ the number of elements in $S$.

1. The values of $r(F R), r(P R P), r(H S)$, and $r(D Y)$ are listed in table 4.4

From Table 4.4 we can see that the new method is more efficient than FR method and PRP method. 


\begin{tabular}{|l|l|l|l|}
\hline FR & PRP & HS & DY \\
\hline 0.775 & 0.896 & 0.923 & 1.212 \\
\hline
\end{tabular}

Table 4.4

\section{References}

[1] Al-Baali, M. Descent property and global convergence of the FletcherReeves method with inexact line search. IMA J. Numer. Anal. 5 (1985), no. 1, 121-124. MR0777963 (86d:49043)

[2] Dai, Yuhong. A nonmonotone conjugate gradient algorithm for unconstrained optimization. J. Syst. Sci. Complex. 15 (2002), no. 2, 139-145. MR1900944 (2003b:90097)

[3] Dai, Y. H.; Yuan, Y. Convergence properties of the Fletcher-Reeves method. IMA J. Numer. Anal. 16 (1996), no. 2, 155-164. doi: 10.1093/imanum/16.2.155

[4] Dai, Y. H.; Yuan, Y. A nonlinear conjugate gradient method with a strong global convergence property. SIAM J. Optim. 10 (1999), no. 1, 177-182. doi $10.1137 /$ S1052623497318992

[5] Fletcher, R.; Reeves, C. M. Function minimization by conjugate gradients. Comput. J. 71964 149-154. MR0187375 (32 \#4827)

[6] Grippo, L.; Lampariello, F.; Lucidi, S. A nonmonotone line search technique for Newton's method. SIAM J. Numer. Anal. 23 (1986), no. 4, 707716. MR0849278 (87g:90105)

[7] Hestenes, M, R., and Stiefel, E, L.: Methods of conjugate gradients for solving linear systems, J. Res. Nat. Bur. Standars Sect., 5 (49), pp. 409436, (1952).

[8] Liu, G, H., Han, J, Y., and Yin, H, X.: Global convergence of the FletcherReeves algorithm with an inexact line search, Appl. math. J. Chinese Univ. Ser. B, 10, pp. 75-82, (1995).

[9] Liu, G. H.; Jing, L. L.; Han, L. X.; Han, D. A class of nonmonotone conjugate gradient methods for unconstrained optimization. J. Optim. Theory Appl. 101 (1999), no. 1, 127-140. MR1685579 
[10] Lucidi, S.; Roma, M. Nonmonotone conjugate gradient methods for optimization. System modelling and optimization (Compiègne, 1993), 206-214, Lecture Notes in Control and Inform. Sci., 197, Springer, London, 1994. MR1294156

[11] Moré, J, J., Garbow, B, S., and Hillstrom, K, E.: Testing unconstrained optimization software, ACM Transactions on mathematical software., 1741, (1981). ACM0098-3500/81/0300-0017

[12] Polyak, B, T.: The conjugate gradient method in extremal problems, Comput. Math. Math. Phys., 9, pp. 94-112, (1969). dx.doi.org/10.1016/00415553(69)90035-4

[13] Powell, M. J. D. Nonconvex minimization calculations and the conjugate gradient method. Numerical analysis (Dundee, 1983), 122-141, Lecture Notes in Math., 1066, Springer, Berlin, 1984. MR0760460.

[14] Zhang, Li; Zhou, Weijun; Li, Donghui. Global convergence of a modified Fletcher-Reeves conjugate gradient method with Armijo-type line search. Numer. Math. 104 (2006), no. 4, 561-572. DOI 10.1007/s00211-006-0028-z 